\title{
Evaluación de competencias digitales de los estudiantes del sector rural y urbano de Chimborazo
}

\section{Evaluation of digital skills of students in the rural and urban sector of Chimborazo}

\author{
Msc. Myriam Elizabeth Murillo Naranjo ${ }^{1}$ \\ Msc. Luis Miguel Viñán Carrasco ${ }^{2}$ \\ Msc Andrés Leandro Rodríguez Galán ${ }^{3}$ \\ Msc. José Vinicio Palacios Carillo ${ }^{4}$
}

UNACH

\section{RESUMEN}

El presente artículo evalúa las competencias digitales de los estudiantes de bachillerato del sector rural y urbano de Chimborazo - Ecuador. Actualmente este tema recupera mayor interés por la emergencia sanitaria que de manera abrupta cambió la modalidad de educación

$1 \quad$ Magíster en diseño y gestión de proyectos socioeducativos, Doctora en Ciencias de la Educación Especialidad Investigación Socio Educativa, Directora de la carrera de Comunicación Social UNACH. myriammurillo@unach. edu.ec. ORCID: https://orcid.org/0000-0002-5141-353X

${ }^{2}$ Magíster en Diseño y Gestión de Marca. Docente de la carrera de Comunicación Social UNACH. luis.vinan@unach. edu.ec ORCID: https://orcid.org/0000-0003-2127-4038

${ }^{4}$ Magister en Gestión de Marketing y Servicio al Cliente. Docente de la carrera de Comunicación Social UNACH. andres.rodriguez@unach.edu.ec ORCID: https://orcid. org/0000-0001-5127-3832

${ }^{4}$ Magíster en Comunicación y Periodismo. Docente de la carrera de Comunicación Social UNACH.jose.palacios@unach.edu.ec ORCID: https://orcid.org/0000-0002-9079-2536 presencial de alrededor de 1300 millones de estudiantes a educación virtual en todo el mundo. Metodológicamente presenta un enfoque cualitativo - cuantitativo. La técnica utilizada es la encuesta, el instrumento el cuestionario; se evaluaron cinco áreas competenciales. La muestra fue de 328 estudiantes del sector urbano y 429 del sector rural de Chimborazo. Entre los principales resultados se evidencia que la conectividad en el sector rural es escasa; en el sector urbano un grupo considerable de estudiantes tiene acceso a internet, es decir, que el acceso a la educación en la actualidad está privatizado para los que tienen este recurso. Las competencias digitales adquiridas por los estudiantes en los dos sectores educativos son desiguales: la competencia más desarrollada es la comunicación en mayor grado en el sector 
urbano, la menos desarrollada es la competencia seguridad en la red; no existe una comprensión cabal de los peligros que implica el excesivo e inadecuado uso de la tecnología en la salud. Finalmente, se identifica que las competencias digitales en los dos grupos fueron adquiridas de manera autónoma y no necesariamente en los centros educativos; el uso de las TIC es de carácter personal e incipiente en procesos educativos. Con estos resultados surge la necesidad de proponer procesos de alfabetización digital para acortar esta brecha digital.

PALABRAS CLAVE: Competencias digitales, Bachillerato, TIC, Procesos de aprendizaje, Alfabetización digital.

\section{ABSTRAC}

This article assesses the digital skills of high school students in the rural and urban sectors of Chimborazo - Ecuador, currently this topic is recovering greater interest due to the health emergency that abruptly changed the modality of face-to-face education of around 1,300 million students to virtual education around the world. Methodologically it presents a quantitative approach, the technique used the survey, the instrument the questionnaire, five competency areas were evaluated; the sample consisted of 328 students from the urban sector and 429 from the rural sector of Chimborazo. Among the main results, it is evident that connectivity in the rural sector is scarce, in the urban sector a considerable group of students have access to the internet, that is, access to education is currently privatized for those who have this resource. The digital skills acquired by students in the two educational sectors are unequal, the most developed competence is communication to a greater degree in the urban sector, the least developed is the network security competence, there is no complete understanding of the dangers that implies the excessive and inappropriate use of technology in health.
Finally, it is identified that digital competences in the two groups were acquired autonomously and not necessarily in educational centers, the use of ICT is personal and incipient in educational processes, with these results the need arises to propose processes of digital literacy to bridge this digital divide.

KEYWORDS: Digital skills, High school, TIC, Learning processes, Digital literacy.

\section{INTRODUCCIÓN}

Actualmente se vive en un contexto de disrupción digital en la Sociedad de la Información y del Conocimiento, en la que las Tecnologías de la Información y la Comunicación (TIC) facilitan la creación, distribución y uso de la información; juegan un papel preponderante en los sectores, educativos, sociales, culturales y productivos. Tienen un desarrollo acelerado e irreversible que vislumbran una revolución digital.

La sociedad presente, denominada digital, está generando nuevas formas de comunicación y de acceso al conocimiento gracias a internet y a los cambios que este fenómeno genera en los medios de comunicación. Los usos y consumos de contenidos audiovisuales, las competencias mediáticas que los ciudadanos deben desarrollar y la relación de las personas entre sí y con las instituciones, son parte de las dinámicas sociales que exigen ser estudiadas para sugerir alternativas que mejoren la calidad de vida, además, son hechos de interés para organismos nacionales e internacionales.

En educación para la ciudadanía mundial, la Unesco (2016) destaca el uso de las tecnologías de la información y de los medios de comunicación como uno de los requisitos esenciales para la formación académica y para la participación social. En un entorno marcado por el desarrollo tecnológico, es necesario que los individuos construyan competencias que 
les permitan desenvolverse de forma eficiente, reflexiva y crítica.

En este marco, la competencia digital juega un rol fundamental, pues además de ser identificada como una de las ocho competencias clave para el aprendizaje permanente (Comisión Europea, 2007), es aquella que permite una utilización óptima de los recursos tecnológicos. Por su parte, los contextos de aprendizaje informal aportan al desarrollo de esta competencia a través de las múltiples posibilidades de interacción con contenidos, personas y herramientas.

Por otra parte, el enfoque de la Agenda Educativa Digital (2017-2021) de Ecuador responde a la instauración de una cultura digital y a las nuevas prácticas de aprendizaje y enseñanza dentro del contexto ecuatoriano de la sociedad del conocimiento. Fomenta en la comunidad educativa competencias digitales, mejoramiento del desempeño, alfabetización digital y participación. Disminuye la brecha digital, en tanto, promueve la generación de contenidos y recursos educativos, acompañados de metodologías innovadoras de enseñanza. Agenda que pronto acabará su proyecto y se evaluarán los resultados.

A esto se suma el importante papel que tienen tanto las competencias digitales como las posibilidades de gestionar los propios aprendiza- jes, pues ambos aportan al "Lifelong Learning", necesario para continuar aprendiendo en diferentes momentos del ciclo vital, aprovechando al máximo lo que se construye en contextos formales, pero también en el ámbito laboral y de ocio (Unesco, 2010).

La competencia digital no se refiere a un concepto unidimensional y estático, sino que implica la articulación de múltiples variables. Instituciones como la International Society for Tecnology in Education (ISTE) han establecido una serie de estándares que permiten evidenciar los distintos componentes de la competencia digital, entre los que se incluyen: creatividad e innovación; comunicación y colaboración; investigación y manejo de información; pensamiento crítico, solución de problemas y toma de decisiones; ciudadanía digital; y conceptos de las TIC (Gutiérrez, Cabero y Estrada, 2017).

Por tal razón, esta investigación se situó desde un enfoque sociocultural y ecológico de la tecnología educativa (Domínguez y Trillo Miravalles, 2014). Desde este enfoque, si bien se reconoce el papel de las tecnologías de la comunicación y de la información en las prácticas de los sujetos, no se asume un determinismo tecnológico, al considerarse que las prácticas se configuran en contextos sociales, a través de la interacción con otros y con elementos propios de la cultura. Desde este enfoque, se concibe que los estudiantes construyen conocimientos tecnológicos en distintos ámbitos y que es necesario favorecer su conexión y validación.

Sobre todo, porque el estudio considera el sector educativo del Bachillerato General Unificado (BGU) que surge de la Ley Orgánica de Educación Intercultural (LOEI) del Ecuador. Es el periodo de estudios, en el que se obtiene el grado de bachiller. Es una enseñanza obligatoria, impartida usualmente desde que el estudiante tiene 15 años, tras haber obtenido la Educación General Básica (BGU), dura tres años. Luego de graduarse de bachiller se puede ingresar en la universidad o a un instituto técnico o tecnológico. EI BGU, que consta de primero, segundo y tercer curso; tiene como triple objetivo preparar a los estudiantes: (a) para la vida y la participación en una sociedad democrática, (b) para el mundo laboral o del emprendimiento, y (c) para continuar con sus estudios universitarios.

Específicamente se analiza el BGU del sector rural y urbano de la provincia de Chimborazo, y de acuerdo al INEC (2010) Chimborazo tiene uno de los porcentajes más altos de población 
indígena en el país el 38\%, mestizos el $56 \%$, blancos el $5 \%$, y mulato $1 \%$. La tasa de analfabetismo en la provincia es del $13,5 \%$. Pero el nuevo mundo digital ha develado esas grietas y lesiones tan profundas en las sociedades y en los países en vías de desarrollo, que estaban no tan visibles antes de la pandemia, eran cifras que el mundo conocía, pero ahora la brecha digital de conectividad presenta de manera frontal a los ricos y pobres por las diferencias de acceso a las tecnologías, que se evidencia en los procesos educativos en toto los niveles educativos del país, nadie estuvo preparado para esta nueva modalidad.

Lo referido anteriormente, concuerda con los que menciona Aguaded, I., \& Contreras-Pulido, P. (2020) hoy más que nunca existe una brecha entre los pueblos que supera las regiones, las razas, las edades y género, las creencias y religiones, la pobreza/riqueza, la cultura y las lenguas... El mundo digital ha generado fisuras estructurales entre inforicos e infopobres, pero que llegan aún más allá, hasta las diferentes formas de afrontar el reto de las tecnologías desde un punto de vista conceptual, actitudinal y valórico. El "empoderamiento digital" supera con creces el reto tecnológico y se adentra en las competencias ciudadanas para vivir un mundo comunicativo en plena era post-COVID-19, en la que se han expandido las potencialidades de la ciber conexión en todas sus dimensiones, de positividad y negatividad para el desarrollo humano.

En tal sentido, el propósito de esta investigación es evaluar las competencias digitales que poseen los estudiantes de bachillerato del sector rural y urbano de Chimborazo - Ecuador, para identificar esa brecha digital entre los dos sectores y proponer procesos de alfabetización digital post- COVID- 2019.
Es importante enfatizar en este punto, que el papel de las TIC es vital en el logro de las competencias digitales, dentro de las competencias básicas que debe poseer todo ciudadano para viabilizar el ejercicio de sus derechos y deberes en sintonía con una sociedad globalizada y cambiante.

El incorporar las TIC en la educación ha traído consigo grandes beneficios a docentes, debido a que facilita la enseñanza de conceptos complejos (Ruiz-Macías \& Duarte,2018; Niño-Vega et al., 2017), así como les permite emplear recursos digitales idóneos según las necesidades de aprendizaje de los estudiantes (Niño-Vega Fernández-Morales \& Duarte, 2019; Jiménez-Espinosa, 2019). Del mismo modo, las TIC favorecen a los estudiantes en el proceso de aprendizaje, ya que permiten adquirir conocimientos de manera más interactiva; les permiten retroalimentar saberes preconcebidos por medio de la experimentación y la gamificación, aumentando el interés y motivación por aprender (Vargas-Vargas, NiñoVega \& Fernández-Morales, 2020; FonsecaBarrera et al, 2020).

Por otra parte, los instrumentos que se utilizaron para la recolección de información fueron la encuestas presenciales ya que se aplicaron en el primer trimestre de 2020, la encuesta se basó en las competencias digitales propuestas por el Marco Común de Competencia Digital Docente (2017) se mantuvo las cinco áreas competenciales, pero las competencias fueron adaptadas a la realidad de los estudiantes de Bachillerato, fueron sometidas a una revisión por dos expertos en el área informática y validadas con el coeficiente Alfa de Cronbach.

Finalmente, no se puede desconocer el esfuerzo actual de los estudiantes, las competencias digitales aprehendidas no necesariamente son adquiridas en las instituciones de educación básica y bachillerato del sector público, ya que la asignatura de computación ya no es parte 
del currículo nacional desde 2015; mientras que en los centros educativos particulares del sector urbano, por lo general sí consideran a esta asignatura con un mínimo de horas, o la utilizan de forma transversal en las demás asignaturas.

\section{MARCO TEÓRICO}

\subsection{Las competencias y competencias digitales}

Las competencias entendidas desde el panorama educativo, según lo manifestado por Marza y Cruz (2018) son asumidas a manera de instrumentos de gran utilidad que permite la movilización de actitudes, conocimientos y procesos; por medio de los cuales los discentes adquieren habilidades para facilitar la transferencia de conocimientos y generar innovación. Por su parte lordache, Mariën y Baelden (2017) proponen que las competencias digitales se asuman como los resultados más prácticos y medibles de los procesos de formación con relación a la novedosa alfabetización digital.

La Comisión Europea (2006, p.15) afirma que la competencia digital es aquella que encamina hacia el uso creativo, seguro y crítico de las TIC, para los diferentes usos de la vida cotidiana. Sin embargo, para poder desarrollar dicha competencia, es necesario el uso apropiado de dispositivos tecnológicos para obtener, evaluar, almacenar, producir, presentar e intercambiar información y comunicarse y participar en redes de colaboración vía internet.

Por otra parte, para poder desarrollar la competencia digital es necesario adquirir conocimientos relacionados con el lenguaje especifico básico alusivo a lo textual, numérico, icónico, visual, gráfico y sonoro (GutiérrezPorlán, Prendes-Espinosa y Martínez- Sánchez, 2018). Se dice que cuando se logra desarrollar competencias digitales, se logra desarrollar destrezas relacionadas a la búsqueda de la información, el uso y manejo de herramientas tic, la creación y curación de contenidos, la seguridad y protección de datos y la capacidad de resolver problemas reales haciendo uso de recursos tecnológicos (MinTIC,2016).

\subsection{Alfabetización digital}

La alfabetización digital es la capacidad de una persona para realizar diferentes tareas en un ambiente digital. Esta definición genérica engloba muchos matices ya que incluiría la habilidad para localizar, investigar y analizar información usando la tecnología, así como ser capaces de elaborar contenidos y diseñar propuestas a través de medios digitales. La alfabetización digital debe entenderse no solo como un medio sino también como una nueva forma de comunicación y de creación y comprensión de la información. (UNIR. 2020).

\section{METODOLOGÍA}

La investigación emplea literatura especializada y documentación actualizada de instituciones como UNESCO, (INTEF) EI Instituto Nacional de Tecnologías Educativas y de Formación del Profesorado (2017); presenta un enfoque cualitativo - cuantitativo, de tipo descriptiva, la principal técnica para la obtención de datos es la encuesta, el instrumento utilizado es el cuestionario, se evalúalas competencias digitales más relevantes para la comunicación online de los estudiantes de Bachillerato General Unificado de centros educativos rurales de Chimborazo y centros educativos urbanos de la ciudad de Riobamba y la importancia para el consumo de información y creación de contenidos digitales, clasificadas en 5 áreas y 19 competencias: 1 . Información (1.1 Navegación, 1.2 Evaluación, 1.3 Almacenamiento); 2. Comunicación (2.1 Interacción, 2.2 Compartir, 2.3 Participación, 2.4 Comportamiento, 2.5 Gestión); 3. Creación de contenidos digitales (3.1 Conocimiento, 3.2.Experiencia, 3.3. Desarrollo, 3.4. Derechos); 4. Seguridad en la red (4.1. Dispositivos, 4.2. Datos, 4.3. Salud, 4.4. Medio ambiente); 5. 
Solución de problemas (5.1. Identificación, 5.2. Resolución, 5.3. Criterios de elección de tecnologías). La escala de medición es de tipo Likert para cada ítem de las competencias, el rango oscila de 1 a 5 puntos, donde $1=$ casi nunca, $2=$ rara vez, $3=$ alguna vez, $4=a$ menudo, $5=$ casi siempre.

Para la selección de la muestra se utilizó el muestreo por conglomerado, estratificado y aleatorio, los datos se obtuvieron de la Dirección Distrital De Educación 06D01 Chambo - Riobamba, lo que permitió definir el número total de estudiantes a encuestar. Para calcular el tamaño de la muestra se utilizó la fórmula de población finita, con un nivel de confianza del $95 \%$, error muestral de $5 \%$, el tamaño de la muestra resultante es de 328 estudiantes de 1ero, 2do, 3ero de Bachillerato General Unificado de instituciones privadas de
Riobamba y 429 estudiantes de bachillerato de instituciones educativas rurales de Chimborazo. La encuesta se desarrolló basada en las competencias digitales propuestas por el Marco Común de Competencia Digital Docente (2017) se mantuvo las cinco áreas competenciales, pero las competencias fueron adaptadas a la realidad de los estudiantes de Bachillerato, fueron sometidas a una revisión por dos expertos en el área informática y validadas con el coeficiente Alfa de Cronbach, se aplicó una prueba piloto a un grupo de estudiantes de Bachillerato y la prueba Alfa de Cronbach arrojó el 0,910 al cuestionario, lo que significa que tiene una confiabilidad alta; después de este proceso se aplicó directamente en las unidades educativas privadas de Riobamba y rurales de Chimborazo, durante los mes de enero y febrero del 2020. Para el procesamiento de datos se utilizó el software SPSS versión 22.

\section{RESULTADOS}

Tabla 1: Datos generales del grupo objetivo estudiantes de Bachillerato General Unificado

\begin{tabular}{|c|c|c|c|c|c|}
\hline \multicolumn{6}{|c|}{ Sector educativo rural y urbano de Chimborazo - Ecuador } \\
\hline \multicolumn{3}{|c|}{$\begin{array}{l}\text { Género - Bachillerato General Unificado sector } \\
\text { rural }\end{array}$} & \multicolumn{3}{|c|}{$\begin{array}{l}\text { Género - Bachillerato General Unificado sector } \\
\text { urbano particular }\end{array}$} \\
\hline Hombres & \multicolumn{2}{|c|}{ Mujeres } & Hombres & \multicolumn{2}{|c|}{ Mujeres } \\
\hline $65 \%$ & \multicolumn{2}{|c|}{$35 \%$} & $49 \%$ & \multicolumn{2}{|c|}{$51 \%$} \\
\hline \multicolumn{3}{|c|}{$\begin{array}{l}\text { Porcentaje de estudiantes del Bachillerato } \\
\text { General Unificado encuestados }\end{array}$} & \multicolumn{3}{|c|}{$\begin{array}{l}\text { Porcentaje de estudiantes del Bachillerato } \\
\text { General Unificado encuestados }\end{array}$} \\
\hline 1 ero & $2 \mathrm{do}$ & 3ero & 1ero & 2do & 3ero \\
\hline $28 \%$ & $39 \%$ & $33 \%$ & $30 \%$ & $37 \%$ & $33 \%$ \\
\hline
\end{tabular}

Fuente: Encuestas aplicadas en el sector urbano y rural en las instituciones de educación- BGU

Serealiza un estudio en dos realidades educativas de la Provincia de Chimborazo, y específicamente el Bachillerato General Unificado en el sector rural y urbano de Chimborazo, considerando que el Bachillerato constituye el tercer nivel de educación escolarizado que continúa y complementa las destrezas desarrolladas en los tres subniveles de Educación General Básica, en el que se evidencia una formación integral e interdisciplinaria vinculada a los valores de justicia, innovación, solidaridad y que permite al estudiante articularse con el Sistema de Educación Superior y, de esta manera, contribuir a su plan de vida. Sin embargo, no habla de las competencias digitales que deben aprender para articularse adecuadamente al aprendizaje. 
Tabla 2: Equipamiento

\begin{tabular}{|l|c|c|c|c|}
\hline Equipo disponible & \multicolumn{2}{|l|}{ Estudiantes de BGU. Urbano } & \multicolumn{2}{l|}{ Estudiantes de BGU. Rural } \\
\hline Indicador & Sí & No & Sí & No \\
\hline Computador de escritorio & $62 \%$ & $38 \%$ & $28 \%$ & $72 \%$ \\
\hline Ordenador portátil & $67 \%$ & $33 \%$ & $19 \%$ & $81 \%$ \\
\hline Wifi & $59 \%$ & $41 \%$ & $20 \%$ & $80 \%$ \\
\hline Teléfono móvil inteligente & $62 \%$ & $38 \%$ & $41 \%$ & $59 \%$ \\
\hline Tablet & $42 \%$ & $58 \%$ & $13 \%$ & $87 \%$ \\
\hline Smart TV & $71 \%$ & $29 \%$ & $16 \%$ & $84 \%$ \\
\hline
\end{tabular}

Fuente: Encuestas aplicadas en el sector urbano y rural en las instituciones de educación- BGU

El acceso de tecnología para estos estudiantes varía evidentemente entre el sector rural y urbano, el equipo de mayor acceso es el celular en los dos casos, claro que la conectividad tiene una diferencia del $41 \%$ en el sector urbano y tan solo el $20 \%$ en el sector rural, lo que significa que, aunque tengan en su mayoría celular esto no garantiza que puedan acceder a la conectividad y al aprendizaje de la misma manera; el equipo que la mayoría no lo tiene son las Tablet.

Tabla 3: Conectividad

\begin{tabular}{|c|c|c|}
\hline Internet & $\begin{array}{l}\text { Bachillerato General } \\
\text { Unificado sector urbano }\end{array}$ & $\begin{array}{l}\text { Bachillerato General } \\
\text { Unificado sector rural }\end{array}$ \\
\hline Alta velocidad & $38 \%$ & $5 \%$ \\
\hline Conexión lenta & $32 \%$ & $15 \%$ \\
\hline No tiene & $30 \%$ & $80 \%$ \\
\hline Lugar de uso & $\begin{array}{l}\text { Bachillerato General } \\
\text { Unificado sector urbano }\end{array}$ & $\begin{array}{l}\text { Bachillerato General } \\
\text { Unificado sector rural }\end{array}$ \\
\hline Casa & $68 \%$ & $21 \%$ \\
\hline Centro de estudios & $20 \%$ & $19 \%$ \\
\hline $\begin{array}{l}\text { Lugares públicos con WIFI abierta: restaurante, } \\
\text { cibercafé, } \\
\text { Infocentro... }\end{array}$ & $12 \%$ & $60 \%$ \\
\hline $\begin{array}{l}\text { Uso de internet en el ámbito personal y } \\
\text { académico }\end{array}$ & $\begin{array}{l}\text { Bachillerato General } \\
\text { Unificado sector urbano }\end{array}$ & $\begin{array}{l}\text { Bachillerato General } \\
\text { Unificado sector rural }\end{array}$ \\
\hline Buscar información para uso personal & $48 \%$ & $36 \%$ \\
\hline $\begin{array}{l}\text { Se relaciona con sus contactos a través de } \\
\text { mensajería instantánea }\end{array}$ & $64 \%$ & $64 \%$ \\
\hline Escucha música y ve películas en streaming & $48 \%$ & $32 \%$ \\
\hline $\begin{array}{l}\text { Usa redes sociales a nivel personal (Facebook, } \\
\text { Instagram, etc.) }\end{array}$ & $61 \%$ & $55 \% \%$ \\
\hline Hace compras online & $39 \%$ & $7 \%$ \\
\hline Participa activamente en foros & $52 \%$ & $13 \%$ \\
\hline Trabajar de manera colaborativa & $36 \%$ & $11 \%$ \\
\hline $\begin{array}{l}\text { Sube y comparte elementos multimedia creados } \\
\text { por usted }\end{array}$ & $31 \%$ & $22 \%$ \\
\hline
\end{tabular}

Fuente: Encuestas aplicadas en el sector urbano y rural en las instituciones de BGU 
Al hablar de conectividad en estos dos grupos de estudiantes se evidencia que el $41 \%$ de estudiantes del sector urbano no tiene internet y el $80 \%$ no lo tiene en el sector rural, casi el doble, sin embargo, parecería que en la ciudad todos deberían tener acceso a internet, pero no es así; en el sector rural solo existe un $5 \%$ de alta velocidad en su internet y el $58 \%$ en el sector urbano.

Al hablar del lugar de uso del internet, la mayoría lo hace en casa en los dos sectores educativos, el $68 \%$ en el sector urbano y el $21 \%$ en el sector rural, pero en los centros educativos es muy bajo su uso para el aprendizaje, el $20 \%$ y el $19 \%$ en el sector urbano y rural respectivamente. Lo que significa que en el currículo de su formación no se contempla como eje fundamental de aprendizaje a las TICs. Por otra parte, los lugares públicos con Wifi abierto son más utilizados en el sector rural ya que existen los Infocentros donde suelen ir a conectarse para socializar y estudiar en un $60 \%$ y en el sector urbano su uso es menor, pero existe en un $12 \%$ por lo general en restaurantes o centros comerciales.
En el indicador, el uso de internet en el ámbito personal y académico se visualiza su mayor utilidad para buscar información de uso personal, es decir, se desperdicia la oportunidad para usos académicos. La mensajería instantánea es la forma de comunicación con sus pares de igual manera en los dos sectores educativos. Escuchan música y ven películas en streaming, en su mayoría lo hacen en el sector urbano, con una diferencia porcentual del $12 \%$ con el sector rural. Usa redes sociales a nivel personal (Facebook, Instagram, etc.) este es el indicar que tiene el segundo lugar de los más utilizados, tan solo con una diferencia porcentual del $6 \%$ en los sectores analizados. Al hablar del indicador, sube y comparte elementos multimedia creados por ellos mismos, no se evidencia mayor producción de contenidos elaborados y compartidos por ellos, en el sector urbano se evidencia una producción del $31 \%$ y en el sector rural el $20 \%$, es decir, una diferencia porcentual de $11 \%$. Lo que casi no se evidencia es la compra vía online en el sector rural, debido al factor socio económico de este sector.

Tabla 4: Estudios y certificados

\begin{tabular}{|c|c|c|}
\hline \multirow{2}{*}{ Indicador } & $\begin{array}{r}\text { Cómo han adquirido los conocimientos actuales sobre Tecnologías de } \\
\text { la Información y la Comunicación }\end{array}$ \\
\cline { 2 - 3 } & $\begin{array}{c}\text { Bachillerato General Unificado } \\
\text { sector urbano }\end{array}$ & $\begin{array}{c}\text { Bachillerato General Unificado } \\
\text { sector rural }\end{array}$ \\
\hline $\begin{array}{c}\text { De manera autodidacta, } \\
\text { (recursos de Internet, } \\
\text { experiencia) }\end{array}$ & $28 \%$ & $15 \%$ \\
\hline $\begin{array}{c}\text { En centros de formación } \\
\text { privados }\end{array}$ & $11 \%$ & $9 \%$ \\
\hline
\end{tabular}

Fuente: Encuestas aplicadas en el sector urbano y rural en las instituciones de BGU

Al considerar el indicador, estudios y conocimientos de Tecnología de la Comunicación e información se observa que en los dos sectores evaluados no hay mayor conocimiento de las TIC, ya que en el sector urbano el $28 \%$ lo hace de manera autodidacta y el $15 \%$ en el sector rural. Al hablar de que, si adquirieron los conocimientos sobre las TIC en centros de formación privados en el sector urbano el $11 \%$ y el $9 \%$ en el sector rural, esto demuestra que en los dos sectores educativos los estudiantes no se preparan en centros especializados sobre el uso de las TIC, el factor económico puede ser un atenuante o porque consideran ser nativos digitales. 
Tabla 5: Análisis de las áreas competenciales y competencias digitales

\begin{tabular}{|c|c|c|c|c|}
\hline $\begin{array}{l}\text { Áreas } \\
\text { Competenciales }\end{array}$ & Competencias & $\begin{array}{l}S \text { e c t o } r \\
\text { u } r \text { b a } n \text { o } \\
\text { BGU } \%\end{array}$ & $\begin{array}{l}\text { S e c t o } r \\
r \text { u } r \text { a } I \\
\text { BGU\% }\end{array}$ & $\begin{array}{l}\text { Diferencia } \\
\%\end{array}$ \\
\hline \multirow{3}{*}{ Información } & $\begin{array}{l}\text { Navegación: Acciones para localizar y } \\
\text { acceder a la información }\end{array}$ & $41 \%$ & 24 & $17 \%$ \\
\hline & Evaluación de la información de internet & $34 \%$ & $23 \%$ & $11 \%$ \\
\hline & Almacenamiento y gestión de documentos & $35 \%$ & $28 \%$ & $7 \%$ \\
\hline \multirow[t]{5}{*}{ Comunicación } & $\begin{array}{l}\text { Interacción a través de: WhatsApp, Telegram, } \\
\text { Messenger, Skype, y redes sociales }\end{array}$ & $65 \%$ & $30 \%$ & $35 \%$ \\
\hline & Comparte información por correo electrónico & $25 \%$ & $16 \%$ & $9 \%$ \\
\hline & $\begin{array}{l}\text { Participa en redes sociales: Facebook, } \\
\text { Twitter, Snapchap, Linkedln e Instagram. }\end{array}$ & $57 \%$ & $28 \%$ & $29 \%$ \\
\hline & $\begin{array}{l}\text { Comportamiento: Interacción respetuosa } \\
\text { con otras personas en Internet }\end{array}$ & $34 \%$ & $24 \%$ & $10 \%$ \\
\hline & $\begin{array}{l}\text { Gestión: Identidad virtual y reputación por } \\
\text { los contenidos que imparte en internet }\end{array}$ & $40 \%$ & $25 \%$ & $15 \%$ \\
\hline \multirow[t]{4}{*}{$\begin{array}{l}\text { Creación de } \\
\text { contenidos } \\
\text { digitales }\end{array}$} & $\begin{array}{l}\text { Conocimiento de herramientas. } \\
\text { Presentaciones: PowerPoint, Impress, } \\
\text { Google Presentaciones }\end{array}$ & $41 \%$ & $19 \%$ & $22 \%$ \\
\hline & $\begin{array}{l}\text { Experiencia en la creación de contenidos } \\
\text { digitales simples }\end{array}$ & $45 \%$ & $23 \%$ & $22 \%$ \\
\hline & $\begin{array}{l}\text { Desarrollo: realiza cambios básicos sobre } \\
\text { los contenidos multimedia: fotografías, } \\
\text { audios y vídeos. }\end{array}$ & $38 \%$ & $20 \%$ & $18 \%$ \\
\hline & Derechos de autor y licencias de uso & $29 \%$ & $10 \%$ & $19 \%$ \\
\hline \multirow[t]{4}{*}{$\begin{array}{l}\text { Seguridad en la } \\
\text { red }\end{array}$} & $\begin{array}{l}\text { Uso seguro de los dispositivos digitales en } \\
\text { Internet }\end{array}$ & $33 \%$ & $24 \%$ & $9 \%$ \\
\hline & $\begin{array}{l}\text { Datos: Interacción en redes sociales, } \\
\text { medidas de seguridad básicas }\end{array}$ & $34 \%$ & $21 \%$ & $13 \%$ \\
\hline & $\begin{array}{l}\text { Actitud y comportamiento al usar las TIC, } \\
\text { repercusión sobre la salud }\end{array}$ & $34 \%$ & $22 \%$ & $12 \%$ \\
\hline & $\begin{array}{l}\text { Comportamiento "ecológico" al usar } \\
\text { tecnología }\end{array}$ & $27 \%$ & $8 \%$ & $19 \%$ \\
\hline \multirow[t]{3}{*}{$\begin{array}{l}\text { Solución de } \\
\text { problemas }\end{array}$} & $\begin{array}{l}\text { Identifica problemas al usar dispositivos } \\
\text { digitales }\end{array}$ & $36 \%$ & $20 \%$ & $16 \%$ \\
\hline & $\begin{array}{l}\text { Resuelve adecuadamente los problemas } \\
\text { que pueden surgir cuando los dispositivos } \\
\text { no funcionan correctamente }\end{array}$ & $26 \%$ & $17 \%$ & $9 \%$ \\
\hline & $\begin{array}{l}\text { Criterios de elección de tecnologías: evalúa y } \\
\text { elige de manera adecuada una herramienta, } \\
\text { dispositivo o servicio para realizar las tareas. }\end{array}$ & $31 \%$ & $17 \%$ & $14 \%$ \\
\hline
\end{tabular}

Fuente: Encuestas aplicadas en el sector urbano y rural en las instituciones de BGU 
Los resultados presentados en esta tabla fueron tomadas de la escala de Likert aplicada, el indicador cinco, es decir, frecuencia siempre.

En la competencia información se evidencia que la mayor diferencia de uso con frecuencia siempre, se encuentra en el indicador navegación: acciones para localizar y acceder a la información, en el sector urbano registra el $41 \%$, en el sector rural $24 \%$, con una diferencia porcentual del $27 \%$.

En la competencia comunicación, registra la diferencia del $35 \%$ en el indicador interacción a través de: WhatsApp, Telegram, Messenger, Skype, y redes sociales, en los centros educativos del sector urbano registra el $65 \%$ y en el sector rural el $30 \%$. Lo que significa que hay interacción con estos recursos, pero en diferente medida por los niveles de conectividad y equipos que poseen.

En la creación de contenidos digitales, dos indicadores tienen el mismo nivel de diferencia porcentual en su uso, en la primera que es el conocimiento de herramientas. Presentaciones: PowerPoint, Impress, Google Presentaciones con una diferencia del $22 \%$, es decir, el sector rural sigue con desventaja; y en la experiencia en crear contenidos digitales simples el $22 \%$ de diferencia, donde el sector urbano tiene más experiencia.

En la competencia seguridad en la red se refiere a cómo los usuarios protegen información y datos personales en contextos digitales, así como el uso responsable que hacen de las tecnologías para el cuidado de la salud y del ambiente, registra el nivel más alto de diferencia entre los dos grupos de estudio, el $19 \%$ en el indicador comportamiento "ecológico" al usar tecnología, a pesar de que su uso es básico para los dos grupos.

En la competencia solución de problemas que asume la capacidad del usuario para identificar sus necesidades en los entornos digitales, evaluar alternativas y tomar decisiones razonadas. En el indicador identifica problemas al usar dispositivos digitales reporta la brecha más notoria entre los dos sectores de estudio del $16 \%$, lo que demuestra la diferencia entre el sector urbano el $36 \%$ y en el sector rural del $20 \%$, esta es una de las competencias que se debe tratar en procesos de alfabetización digital para que los equipos no queden obsoletos 0 subutilizados.

\section{CONCLUSIONES:}

En relación al equipamiento con el que cuentan los estudiantes de Bachillerato General Unificado del sector rural, se evidencia que gran parte de ellos carecen de los equipos básicos como computador de escritorio, portátil, Tablet y solo un grupo tiene acceso a teléfonos móviles inteligentes. Por otra parte, los estudiantes de Bachillerato General Unificado del sector urbano tienen acceso a la mayoría de estos equipos y en menor medida las Tabletas. Estos datos demuestran la brecha digital de acceso a internet en los dos grupos, pero los menos favorecidos por la situación socioeconómica y geográfica en el sector rural.

En lo que se refiere a conectividad en el sector rural la mayoría no tiene acceso a internet, en el sector urbano un grupo considerable de estudiantes tienen acceso a internet, lo que significa que el acceso a la educación en la actualidad está privatizado para los que tienen este recurso.

Al analizar el uso de los equipos e internet en el sector rural lo hacen en su mayoría en los Infocentros comunitarios, que son una de las metas cumplidas de la estrategia Ecuador Digital 2.0 para las parroquias rurales, que son espacios de participación y encuentro en los que se garantiza el acceso a las Tecnologías de la Información y Comunicación (TIC), que contribuyen a la reducción de la brecha digital, porque el acceso a internet es escaso o no 
existe en estos lugares; se utiliza en menor grado este recurso en los centros de estudio. En el sector urbano existe un alto nivel de uso de internet en el hogar y lugares públicos, pero bajo su nivel en los centros de estudio.

Al evaluar las competencias digitales que poseen los estudiantes del sector urbano y rural se evidenció lo siguiente:

En el área competencial digital información en la competencia navegación es donde existe una diferencia mayor entre el sector rural y urbano en las acciones para localizar y acceder a la información, los estudiantes del sector urbano lo hacen con mayor facilidad.

En el área competencial comunicación en la competencia interacción, es donde registra la mayor actividad de los dos sectores objeto de estudio a través de: WhatsApp, Telegram, Messenger, Skype, y redes sociales, en el sector rural en menor medida. También tienen un buen nivel de interacción en la competencia participación en redes sociales: Facebook, Twitter, Snapchap, Linkedln e Instagram, el sector urbano en mayor medida y en el sector rural en menos proporción, utilizan esta competencia para actividades personales, para actividades educativas es muy escasa en los dos grupos.

En el área competencial creación de contenidos digitales, la competencia experiencia en la creación de contenidos digitales simples, los estudiantes del sector urbano tienen un nivel medio y los del sector rural un nivel básico. Tienen un conocimiento incipiente en los dos grupos sobre los derechos de autor y licencias de uso.

Al evaluar el área competencial seguridad en la red, en la competencia actitud y comportamiento al usar las TIC, repercusión sobre la salud los estudiantes del sector urbano poseen un conocimiento medio, en le sector rural es bajo, esto implica que no existe una comprensión cabal de los peligros que implica el excesivo e inadecuado uso de la tecnología en la salud de los estudiantes.

En el área competencial solución de problemas, en la competencia identifica problemas al usar dispositivos digitales, los estudiantes del sector urbano resuelven problemas técnicos no complejos relacionados con los dispositivos, mientras que los estudiantes del sector rural identifican un problema técnico básico y explican en qué consiste el mal funcionamiento, pero no siempre lo arreglan.

Finalmente, un proceso de alfabetización digital debe administrarse para estos dos sectores educativos, con base a los resultados obtenidos en la evaluación de las cinco competencias y reforzar las competencias menos desarrolladas para que estas sirvan de herramientas valiosas para el aprendizaje y así acceder a la sociedad del conocimiento empleando las tecnologías de la información y comunicación, impulsando prácticas pedagógicas con enfoque digital que renueven las prácticas de enseñanza y procesos de aprendizaje, para fortalecer el desarrollo y potenciar habilidades de los estudiantes y docentes.

\section{REFERENCIAS BIBLIOGRÁFICAS}

Aguaded, I., \& Contreras-Pulido, P. (2020). Acceso universal y empoderamiento digital de los pueblos frente a la brecha desigual. Nuevas formas de diálogo y participación. Trípodos, (46), 9-12.

Comisión Europea. (2007). Competencias clave para el aprendizaje permanente un marco de referencia europeo. Luxemburgo: Oficina de Publicaciones Oficiales de las Comunidades Europeas.

Domínguez, D. y Trillo Miravalles, P. (2014). Learning Competences in Open Mobile Environments: A Comparative Analysis Between 
Formal and Non-Formal Spaces. Open Praxis, 6(3), 235-244.

Fonseca-Barrera, C. C., Niño-Vega, J. A., \& Fernández-Morales, F. H. (2020). Desarrollo de competencias digitales en programación de aplicaciones móviles en estudiantes de noveno grado a través de tres estrategias pedagógicas. Revista Boletín Redipe, 9(4), 179-191. https:// doi.org/10.36260/rbr.v9i4.958.

Gutiérrez Castillo, J.J., Cabero Almenara, J. y Estrada-Vidal, L.I. (2017). Diseño y validación de un instrumento de evaluación de la competencia digital del estudiante universitario. Revista Espacios, 38(10).

Gutiérrez - Porlán, I., Prendes-Espinosa, M. P., \& Martinez-Sanchez, F. (2018). Competencia digital una necesidad del profesorado universitario en el siglo XXI. RED Revista de Educación a Distancia, 56(7), 1-22. DOI:http:// dx.doi.org/10.6018/red/56/7

Instituto Nacional de Estadística y Censo (INEC) (2010)- Ecuador

Instituto Nacional de Tecnologías Educativas y de Formación del Profesorado - INTEF. (2017b). Marco Común de Competencia Digital Docente. España: Gobierno de España.

lordache, C., Mariën, I., \& Baelden, D. (2017). Developing Digital Skills and Competences: A QuickScan Analysis of 13 Digital Literacy Models. Italian Journal of Sociology of Education, 9(1), 6-30. doi: https://doi.org/10.14658/pupjijse-2017-1-2

Jiménez-Espinosa, A. (2019). La dinámica de la clase de matemáticas mediada por la comunicación. Revista de Investigación, Desarrollo e Innovación, 10 (1), 121-134.

Marza, M., \& Cruz, E. (2018). Gaming como Instrumento Educativo para una Educación en competencias Digitales desde los Academic
Skills Centres. Revista General de Información y Documentación, 28(2), 489-506. Doi: http:// dx.doi.org/10.5209/RGID.60805

Ministerio de Educación del Ecuador (2017). Agenda Educativa Digital (2017-2021). Educación integral para la sociedad del conocimiento y la cultura digital. Quito-Ecuador.

Ministerio de Tecnologías de la Información y Comunicaciones. (2016), Currículos exploratorios, MINTIC. Recuperado de: http://aprende.colombiaaprende.edu.co/es/ curriculostic.

Niño-Vega, J. A., Martínez-Díaz, L. Y., Fernández-Morales, F. H.,Duarte, J. E., ReyesCaballero, F., \& Gutiérrez-Barrios, G. J. (2017). Entorno de aprendizaje para la enseñanza de programación en Arduino mediado por una mano robótica didáctica. Revista Espacios, 38 (60), 23. Recuperado de: http://www.revistaespacios. com/a17v38n60/17386023.html

Niño-Vega, J. A., Fernández-Morales, F. H., \& Duarte, J. E. (2019). Diseño de un recurso educativo digital para fomentar el uso racional de la energía eléctrica en comunidades rurales. Saber, Ciencia y Libertad, 14 (2), 256272. doi: https://doi.org/10.18041/2382-3240/ saber.2019v14n2.5889

Ruiz-Macías, E., \& Duarte, J. (2018). Diseño de un material didáctico computarizado para la enseñanza de Oscilaciones y Ondas, a partir del estilo de aprendizaje de los estudiantes. Revista de Investigación, Desarrollo e Innovación, 8(2), 295-309

Unesco. (2016). Educación para la Ciudadanía Mundial. Preparar a los educandos para los retos delsigloXXI.París. Recuperadodehttp://unesdoc. unesco.org/ images/0024/002449/244957s.pdf.

Unesco. (2010). Belém Framework for Action: Harnessing the power and potential of adult learning and education for a viable future. 
Hamburg, Germany: CONFITEA VI.

Vargas-Vargas, N. A., Niño-Vega, J. A., \& Fernández-Morales, F. H. (2020). Aprendizaje basado en proyectos mediados por tic para superar dificultades en el aprendizaje de operaciones básicas matemáticas. Revista Boletín Redipe, 9(3), 167-180. https://doi. org/10.36260/rbr.v9i3.943 